\title{
Reseña de La santa Juana y el mundo de lo sagrado, ed. Blanca Oteiza, Madrid/New York/Pamplona, Instituto de Estudios Tirsianos/IDEA, 2016, 309 pp., ISBN 978-1-938795-30-5
}

\section{Álvaro Rosa Rivero}

Universidad Internacional de La Rioja

ESPAÑA

alvaro.rosa@unir.net

[Hipogrifo, (issn: 2328-1308), 6.1, 2018, pp. 739-743]

Recibido: 18-09-2017 / Aceptado: 09-10-2017

DOI: http://dx.doi.org/10.13035/H.2018.06.01.53

El Instituto de Estudios Tirsianos del GRISO de la Universidad de Navarra, en colaboración con la Fundación Instituto Castellano y Leonés de la Lengua y el Instituto de Estudios Auriseculares (IDEA), presenta esta interesante monografía dedicada a sor Juana de la Cruz y el mundo de lo sagrado, resultado en parte de la edición de la trilogía tirsiana La santa Juana, que centra el Proyecto Edición crítica del teatro completo de Tirso de Molina. Cuarta fase (referencia FFI2013-48549-P) subvencionado por el Ministerio de Economía y Competitividad del Gobierno de España.

Como señala Blanca Oteiza en la presentación, las contribuciones se disponen en tres bloques temáticos: el primero de ellos está formado por «seis trabajos dedicados a la biobibliografía de sor Juana, tanto de los documentos y circunstancias relativos a su vida como de sus recreaciones literarias, con especial atención a la del Mercedario, ordenados de lo general a lo particular»; en el segundo bloque «se reflexiona desde diversas perspectivas sobre el mundo de lo sagrado o la sacralización en la obra de Tirso, especialmente a partir de sus protagonistas, mayoritariamente femeninas, pero no solo» (p. 9), y el tercero se ocupa «de diversos temas, motivos y personajes de carácter religioso en la obra de autores contemporáneos del Mercedario. Cierra el volumen una actualizada mirada sobre la muerte de Tirso en Almazán» (p. 9).

El primer bloque, dedicado a la santa, se inicia con el estudio de Oteiza, «Documentos y recreaciones de sor Juana. El libro de la casa», que recoge las fuentes biográficas de Juana Vázquez (Vida y fin de la bienaventurada virgen Sancta Juana, 
atribuida a sor María Evangelista); su obra, conocida como El libro del conhorte, colección de 72 sermones que la religiosa pronunciaba cuando se encontraba en éxtasis; las recreaciones literarias de que es protagonista (por ejemplo la trilogía tirsiana La santa Juana) y finalmente El libro de la casa, un curioso manuscrito inédito que contiene materiales diversos «para uso interno de las monjas» del convento: es decir, «las representaciones dramáticas que se realizaban en el convento [...], algunas de las poesías que se cantaban en diversas festividades y fragmentos de la vida y visiones de sor Juana, e indicaciones sobre cómo celebrar algunas festividades» (pp. 18-19). Oteiza se detiene en este manuscrito del que publica las poesías exentas que contiene, como anticipo de la edición que está realizando del Libro de la casa. En el segundo trabajo, titulado «Sor Juana de la Cruz: silencios y modificaciones de la biografía barroca», María Luengo compara las dos biografías canónicas de la santa, la del franciscano fray Antonio Daza publicada en 1610 y 1613, y la del padre Navarro, que se imprimirá en 1622. Luengo pone el acento en las diferencias existentes entre ambas obras, así como en la influencia de la censura en el texto de Daza y, como consecuencia de esto, las modificaciones que realiza Navarro en algunos acontecimientos especialmente comprometidos de la vida de la religiosa. A continuación Isabel Ibáñez en «El entramado teológico-religioso de La santa Juana de Tirso de Molina» analiza los elementos biográficos de Juana de la Cruz presentes en la trilogía tirsiana en relación con el discurso doctrinal e ideológico del que se nutre la obra del Mercedario y la herencia biográfica que estas comedias mantienen con La vida daziana, publicada poco antes de la composición de La santa Juana. Jesús María Usunáriz, por su parte, realiza en «Entre la santidad y la heterodoxia: visionarias en el Tribunal de Logroño (1570-1700)» una interesante investigación sobre las únicas mujeres visionarias que fueron juzgadas ante el tribunal inquisitorial de Logroño durante los años 1570 a 1700, que pone de manifiesto la existencia en España de una «cultura común de las visiones» (p. 78) que era vigilada con recelo por la Inquisición y que formaba parte del imaginario colectivo que Tirso pudo conocer cuando escribió La santa Juana. Muy sugestivo es el trabajo de Alan Paterson en el que analiza los distintos acontecimientos biográficos que componen cada comedia de esta trilogía, así como los diversos recursos escénicos que emplea Tirso para llevar estos hechos a las tablas y convertir este personaje histórico en teatral. Gabriel Téllez logra sintetizar -desde una perspectiva humana, creativa y escénica- los elementos más relevantes de la biografía de sor Juana de la Cruz. Cierra este primer bloque la contribución de Concepción Martínez Pasamar y Cristina Tabernero, dedicada a la lengua femenina de Juana Vázquez y el tratamiento que Tirso realiza de su lengua en La santa Juana. Para tal propósito, se sirven de El libro del conhorte, supuestamente redactado por una mujer que transcribe los sermones de sor Juana cuando se encuentra en éxtasis. Martínez y Tabernero evidencian la destreza de Tirso en la construcción del discurso femenino, manejando hábilmente los recursos orales ligados a la expresividad de la mujer.

La segunda sección de la monografía, centrada en el mundo de lo sagrado en la obra de Tirso de Molina, se articula en ocho capítulos. El primero está escrito por Ignacio Arellano que analiza «la figura de María de Molina en la comedia La 
prudencia en la mujer». Se trata de un agudo capítulo en el que se constata cómo la mitificación de la santidad de la reina -identificada con la Virgen María- posee puntos dudosos que no han sido estudiados por la crítica. Arellano, basándose en el episodio del castigo del médico Ismael, demuestra el marcado antisemitismo de María de Molina (y de Tirso), que ofrece una imagen poco dada a la compasión y a la misericordia. Tirso, aunque intenta realzar la santidad de la reina, ofrece cierto desbarajuste a la hora de constatar este aspecto en el desarrollo de la acción dramática, motivado especialmente por su marcado antisemitismo. Isabelle Bouchiba se ocupa a su vez de la presencia de lo sagrado femenino en siete comedias hagiográficas donde se propone a una mujer como modelo de santidad: - La santa Juana, La ninfa del cielo, Doña Beatriz de Silva, Quien no cae, no se levanta, La joya de la montaña y Los lagos de san Vicente- así como las tres comedias de motivo bíblico donde la figura femenina es también protagonista: - La mejor espigadera, La mujer que manda en casa y La venganza de Tamar-. Tirso establece una «clara y muy sutil jerarquía teológica entre las figuras del Primer Testamento y las santas que emanan del Segundo» (p. 154). María Paloma Fanconi añade otra perspectiva génerica al analizar en «Las santas de Tirso: del teatro y la prosa» la presencia de lo femenino en ambos géneros cultivados por el Mercedario (en comedias como La santa Juana, Los lagos de san Vicente, Quien no cae no se levanta... y en novelas como La patrona de las musas) y observa su importancia en su trayectoria dramática en tanto que Tirso inició «su obra con vidas de santos y la cerró con vidas de santos, en distintas formas, en diversos géneros y de varios modos, en una singladura que, creo, es un botón de muestra del desarrollo y evolución creativa de uno de nuestros grandes del Siglo de Oro» (p. 167). Miguel Galindo analiza La elección por la virtud, comedia que dramatiza la biografía de Félix Pereto, futuro pontífice romano, y la compara con La santa Juana -en la que se constata una mayor presencia de lo sobrenatural y los sucesos milagrosos están representados con mayor verosimilitud-. De esta forma, en la comedia referente a Sixto $v$ Tirso ofrece al espectador una mayor ambigüedad del milagro y de la veracidad de lo sobrenatural. Tanto en La elección por la virtud como en La santa Juana, Tirso lleva a las tablas personajes no canonizados por la Iglesia. «La sacralización del niño en algunas comedias de Tirso de Molina» es el tema en que se centra seguidamente Naïma Lamari. El tratamiento dramático que Tirso realiza de la figura del niño se estudia en dos comedias bíblicas (La mejor espigadera y La vida y muerte de Herodes), en las que se revela similitudes entre los niños protagonistas y el Niño-Dios, y en dos tragedias tirsianas (Todo es dar en una cosa y Escarmientos para el cuerdo) con las que se establecen evidentes paralelismos y similitudes. Philippe Meunier estudia con perspicacia en «Aporía del personaje del galán o cómo dramatizar mejor lo sagrado», la figura de santa Casilda y del galán don Tello en la comedia hagiográfica Los lagos de san Vicente, en la que se «aprovecha dramatúrgicamente» de manera especial el espacio natural de la Bureba burgalesa, a la vez que se cuestiona la figura de este galán «escindido entre la relación mundana con su dama, doña Blanca, y lo imperioso e indecible de su fe cristiana» (p. 200). Elena Nicolás en «Personajes de las comedias hagiográficas de Tirso de Molina. Análisis comparativo» realiza un amplio análisis de las comedias hagiográficas tirsianas (DOÑA BEATRIZ DE Silva, El árbol del mejor fruto, El caballero de Gracia, El mayor desengaño, La dama del 
Olivar, La elección por la virtud, La joya de las montañas, La ninfa del cielo, La Peña de Francia, La santa Juana...), que demuestra algunas particularidades como que «el Mercedario compusiera más comedias hagiográficas de santas que de santos» o que «la Orden de la que Tirso elige más protagonistas para sus comedias hagiográficas es la franciscana, por la que sintió siempre una gran cercanía y simpatía» (p. 221). Cierra este segundo bloque sobre la obra tirsiana el trabajo de Victoriano Roncero («El hombre virrey del mundo: Religión y política en los autos sacramentales de Tirso») en el que analiza la vinculación existente entre religión y política en sus autos sacramentales. Esta implicación trata de constatarse a partir de la influencia de la figura del privado del rey en los autos Los hermanos parecidos y No le arriendo la ganancia.

La última sección del libro amplía la temática al «mundo religioso en autores contemporáneos» con tres artículos de especial interés. El primero, titulado «La misa, de Lutero a Lope: doctrina y paradigmas compositivos», tiene dos partes. Una a cargo de Enrique Duarte en la que analiza tres autos sacramentales de Lope que se escribieron y representaron en la época de La santa Juana (El viaje del Alma, El Tusón del rey del cielo y El misacantano) y el carácter dramático de la Santa Misa que comparten y su valor doctrinal, destinado a popularizar los conceptos de «sacrificio» y «transustanciación» que rechaza Lutero. Y en la segunda, Pablo Blanco detalla, desde el ámbito de la teología católica y protestante, estas nociones eucarísticas. Lara Escudero analiza en «Comicidad (y horror) en el teatro: el motivo de El niño de la Guardia» la comicidad y el horror en tres piezas hagiográficas escritas por autores diferentes en épocas distintas que «tienen en común el argumento histórico-legendario de la profanación de una hostia consagrada y el secuestro y martirio de un niño por los judíos a imitación de la Pasión de Cristo» (p. 266): el auto El mayor desengaño -atribuido a Tirso-, El niño inocente de La Guardia -de Lope de Vega - y La viva imagen de Cristo - de Hoz y Mota Cañizares-. Escudero constata que la comicidad en las comedias hagiográficas no siempre queda relegada a un segundo plano, y que la violencia puede dramatizarse junto con aportaciones cómicas. Teresa Rodríguez cierra esta sección con «La evolución de un patrón de santidad: la figura de san José trazada por Mira de Amescua», un estudio de la figura de san José en el Coloquio del Nacimiento de Nuestro Señor de Mira de Amescua que completa pormenorizadamente con la tradición de la figura josefina en la literatura española anterior y la dramatización del Santo Patriarca que se realiza en el Siglo de Oro.

Esta interesante y completa monografía concluye con un capítulo, a modo de epílogo, en el que José Ángel Márquez revisa la información sobre los últimos días de Tirso y su fallecimiento en Almazán (Soria), contextualizándolos en el Almazán de su época. Tirso fue supuestamente enterrado en el convento mercedario de esta localidad, aunque sin embargo, aún no se han encontrado allí sus restos mortales.

En definitiva, La santa Juana y el mundo de lo sagrado es un provechoso volumen, eficazmente estructurado en su temática, que parte del estudio de la figura de Juana Vázquez -conocida como sor Juana de la Cruz o la santa Juana-, desde distintas perspectivas complementarias: biográficas, literarias, teológicas, etc.; 
continúa con el análisis de lo sagrado en la obra de Tirso de Molina y finaliza con el examen de lo religioso en otros autores contemporáneos. De manera que el libro permite trazar un marco de máximo interés y utilidad para comprender la hagiografía barroca española en la literatura del Siglo de Oro. 